Pacific Journal of Mathematics

ANALYTIC LINEARIZATION OF THE KORTEWEG-DE VRIES
EQUATION (E. 


\title{
ANALYTIC LINEARIZATION OF THE KORTEWEG-DE VRIES EQUATION
}

\author{
E. TAFLIN
}

We prove that the $K d V$ equation is linearized by an analytic function, which is projectively analytically invertible. The Cauchy problem for the $K d V$ equation is entirely solved by this fact. The non-linear superposition principle is a trivial consequence of convexity for the image of the linearization operator.

1. Introduction. Since the discovery [7] of the inverse scattering formalism for the $K d V$ equation

$$
\begin{aligned}
\frac{\partial}{\partial t} u(t, x)+\frac{\partial^{3}}{\partial x^{3}} u(t, x)-6 u(t, x) \frac{\partial}{\partial x} u(t x) & =0, \\
t, x, u(t, x) & \in \mathbf{R},
\end{aligned}
$$

it is known, given a certain class of solution for the linear equation

$$
\frac{\partial}{\partial t} v(t, x)+\frac{\partial^{3}}{\partial x^{3}} v(t, x)=0
$$

how to construct solutions of equation (1.1). However, it is not clear how this reduces the Cauchy problem for the $K d V$ equation, on a given space of initial conditions, into that of the above linear equation. The Cauchy problem for (1.1) has been solved by direct functional analysis methods on the Sobolev space $H^{3},([\mathbf{1 1}])$, and on $H^{2},([\mathbf{1}])$. The inverse scattering formalism has been used to solve the Cauchy problem on $S(\mathbf{R}),([12])$ and for sufficiently rapidly decreasing $C^{3}$ initial conditions ([3]), where in both cases, two linear problems are associated with the $K d V$ equation.

To formulate the problem of linearization of the non linear Cauchy problem, it is convenient to give topological vectorspaces of initial conditions and of solutions for the non linear resp. for the linear problem. In this context it is possible to give a precise meaning to the concept of linearization (see [5]). What we want to show in this paper is that the linearization program defined in [6], entirely goes through and solves the initial value problem for the $K d V$ equation. We stress the fact that this approach is straightforward (in contrast to the inverse scattering formalism), when the spaces of initial conditions are given. The inverse 
scattering formalism is recovered (c.f. [8]). This is not surprising as, with the particular choice of spaces we have done, the linearization is unique (for fixed first term).

The choice of spaces is of course important for the properties of the linearization mapping (if it exists). It is easy to illustrate this fact for the $K d V$ equation. Let $E_{1}$ and $E_{2}$ be two TVS of functions $f: \mathbf{R} \rightarrow \mathbf{R}$ and $F$ : $E_{1} \rightarrow E_{2}$ a $C^{1}$, one to one map, which linearizes the $C^{0}$ vector fields on $E_{1}$ :

$$
\begin{gathered}
X_{0}(u)=\partial u, \quad X_{1}(u)=-\partial^{3} u+6 u \partial u, \\
{[\partial u](x)=\frac{\partial}{\partial x} u(x), \quad u \in E_{1},}
\end{gathered}
$$

i.e. $F$ is a translation invariant linearization of the $K d v$ equation. Denoting $D$ the Frechet derivative, this means that

$$
\text { DF. } X_{0}=X_{0}^{1} \circ F, \quad D F . X_{1}=X_{1}^{1} \circ F,
$$

where $X_{1}^{1}(u)=-\partial^{3} u$ and $X_{0}^{1}(u)=X_{0}(u)$. Let $u_{0} \neq 0$ be the initial condition for a soliton, i.e.

$$
k X_{0}\left(u_{0}\right)=X_{1}\left(u_{0}\right) \text { for some } k<0 \text {. }
$$

Then

$$
0=D F .\left(k X_{0}-X_{1}\right)\left(u_{0}\right)=\left(k X_{0}^{1}-X_{1}^{1}\right) \circ F\left(u_{0}\right)
$$

i.e. $\left(k \partial+\partial^{3}\right) v_{0}=0$ for $v_{0}=F\left(u_{0}\right)$

The non-constant solutions of this equation in $D$ are exponentials. Thus if $F$ is one to one and is defined on the solitons then $E_{2}$ has to contain exponentials. Further if $F^{-1}$ exists as a $C^{1}$ function on some open set $O \ni 0$ in $E_{2}$ then $D F(0)$ and $D F(0)^{-1}$ are continuous linear mappings. So $E_{1}$ and $E_{2}$ are topologically isomorphic. One can then as well choose $E_{1}=E_{2}=E$. Suppose that $C^{\infty}(\mathbf{R}) \supset E \supset S(\mathbf{R})$ (the Schwartz space of test functions) and that $E$ contains at least the exponentials $e^{a x}, a>0$. If $F$ is $C^{\infty}$, one finds by calculating (in a very formal way) some of the derivatives $D^{n}\left(F^{-1}\right)$, (see [8] for explicit formulas) that the operator $f \rightarrow \partial f$ has to be invertible on $E$, i.e. $f(x) \rightarrow 0$ fast as $x \rightarrow-\infty$.

These qualitative remarks show that it is a quite natural choice to treat the linearization problem for the $K d V$ equation on a space like the TVS of all functions $f \in C^{\infty}(\mathbf{R})$ for which the seminorms

$$
\|f\|_{N}=\sup _{\substack{x \in]-\infty, N] \\ 0 \leq k \leq N}}\left|(1+|x|)^{N} \partial^{k} f(x)\right|, \quad N=0,1, \ldots,
$$


are finite. On this space, denoted $S_{b}$, we prove (Corollary 3.3 and Proposition 3.4) that there is a unique translation invariant formal linearization $A$ (taking the non linear equation into the linear) of the $K d V$ equation (in the sense of [5]), i.e. the linearization operator exists as a formal power series where each term is finite and commute with the space translations. $A$ converges to an entire analytic function $\hat{A}: S_{b} \rightarrow S_{b}$ (Corollary 4.2). The power series $A^{-1}$ defines a $C^{\infty}$ mapping $\hat{A}^{-1}: \hat{A}\left[S_{b}\right] \rightarrow$ $S_{b}$, which is "projectively analytic" (Corollary 4.4 and Proposition 4.6). The set $\hat{A}\left[S_{b}\right]$ is convex, which gives the non-linear superposition principle for solutions of the $K d V$ equation. The Cauchy problem for the $K d V$ equation can now be solved entirely by linearization (Proposition 5.2 and Remark 5.3).

2. Formal linearization. Let us first introduce some necessary notation. Given two $T V S, X$ and $Y$. Denote $F(X, Y)\left(\right.$ resp. $\left.F_{s}(X, Y)\right)$ the space of formal power series from $X$ to $Y$ of the form $f=\Sigma_{n \geq 1} f^{n}$, where $f^{n} \in \mathcal{L}_{n}(X, Y)$ (resp. $\mathcal{L}_{n}^{s}(X, Y)$ ), the space of $n$-linear continuous (resp. continuous symmetric) mappings from $X$ to $Y . f_{s} \in F_{s}(X, Y)$ will denote the symmetrization of $f \in F(X, Y)$. If $X, Y$ and $Z$ are $T V S$ then the product

$$
F_{s}(Y, Z) \times F_{s}(X, Y) \ni(A, B) \mapsto A * B \in F_{s}(X, Z)
$$

is defined by (see [5]):

$$
A * B=\sum_{n \geq 1}\left(\sum_{1 \leq p \leq n} A^{p}\left(\sum_{0 \leq q \leq p-1} I_{q} \otimes B^{n-p+1} \otimes I_{p-q-1}\right) \sigma_{n}\right)
$$

where $I_{q}$ is the identity mapping on $X \hat{\otimes}_{s} \cdots \hat{\otimes}_{s} X$ ( $q$-times) and $\sigma_{n}$ is the normalized symmetrization mapping on $\hat{\otimes}^{n} X$. $\hat{\otimes}_{s}$ is the symmetric projective tensor product. $F(X, X)$ is denoted $F(X)$, etc. If $A, B \in F_{s}(X)$ then the bracket $[A, B]_{*}=A * B-B * A$ is defined.

Remark 2.1. If $A, B \in F_{s}(X)$ define entire functions $\hat{A}, \hat{B}: X \rightarrow X$, where

$$
\hat{A}(u)=\sum_{n=1}^{\infty} \hat{A}^{n}(u), \quad \hat{A}^{n}(u)=A^{n}(u, \ldots, u)
$$

then

$$
D \hat{A} \cdot \hat{B}=\widehat{A * B} \text { and }[\hat{A}, \hat{B}]=[\widehat{A, B}]_{*} \text {. }
$$

Here $D$ is the Frechet derivative and $[\hat{A}, \hat{B}]=D \hat{A} . \hat{B}-D \hat{B} \cdot \hat{A}$ the usual vector field bracket. 
We denote by $S_{b}$ the Frechet space of all $C^{\infty}$ functions $f$ from $\mathbf{R}$ to $\mathbf{R}$ for which $\|f\|_{N}<\infty, N=0,1, \ldots$. The seminorms in $S_{b}$ are given by

$$
\|f\|_{N}=\sup _{\substack{x \in 1-\infty, N] \\ 0 \leq k \leq N}}\left|(1+|x|)^{N} \partial^{k} f(x)\right|, \quad[\partial f](x)=\frac{\partial}{\partial x} f(x) .
$$

$S_{b}$ is the projective limit of the spaces $S(n), n=0,1, \ldots$ where $S(n)$ is the subset of all $\left.\left.f \in C^{\infty}(]-\infty, n\right]\right)$ such that

$$
\sup _{\substack{x \in]-\infty, n] \\ 0 \leq \alpha \leq k}}\left|(1+|x|)^{k} \partial^{\alpha} f(x)\right|<\infty, \quad k=0,1, \ldots,
$$

Proposition 2.1. The Frechet space $S_{b}$ is a nuclear Montel space.

Proof. $S_{b}$ is barreled as it is Frechet. Let $B \subset S_{b}$ be a bounded closed subset, i.e. $\|B\|_{N}=\sup _{f \in B}\|f\|_{N}<\infty$ for each $N \geq 0$. It follows from the Ascoli-Arzela theorem that if $\|B\|_{N+1}<\infty$, then for each sequence $\left\{f_{n}\right\}_{n=0}^{\infty}$ in $B$ there exists a subsequence $\left\{f_{i_{n}}\right\}_{n=0}^{\infty}$ such that $f_{i_{n}}$ converges in the \|\|$_{N}$ norm. By a diagonalisation argument there is then a subsequence $\left\{f_{j_{n}}\right\}_{n=0}^{\infty}$ of $\left\{f_{n}\right\}_{n=0}^{\infty}$ which converges in the norm \|\|$_{N}$ for each $N \geq 0$. This proves that $S_{b}$ is a Montel space.

That $S_{b}$ is a nuclear space from the fact that $S$ is nuclear. In fact since $S$ is nuclear, the space $S(n)=S / E_{n}, n \geq 0$, is nuclear (c.f. [9] Theorem III.7.4), where $E_{n}$ is the closed subspace of functions $f \in S$ such that supp $f \subset\left[n, \infty\left[. S_{b}\right.\right.$, being the projective $\operatorname{limit} \lim S(n)$, is then nuclear (cf. [9] corollary of Theorem III.7.4).

Let $E_{N}$ be the closed subspace of $S^{\prime}$ of distributions with support in ]$-\infty, N]$.

Proposition 2.2. $S_{b}^{\prime}$ (the strong dual of $S_{b}$ ) is a nuclear Montel space, and is isomorphic to the strict inductive limit of $\left\{E_{N}\right\}_{N=0}^{\infty}$.

Proof. As $S$ is dense in $S_{b}, S_{b}^{\prime} \subset S^{\prime}$ (set theoretically). Further if $T \in S^{\prime}$ has a continuous extension to $S_{b}$ then $\left.\left.\operatorname{supp} T \subset\right]-\infty, N\right]$ for some $N \geq 0$. Each semi norm in $S_{b}^{\prime}$ has the form $S_{b}^{\prime} \ni F \rightarrow q_{B}(F)=$ $\sup _{u \in B}|F(u)|$, where $B$ is a bounded set in $S_{b}$. The inductive limit of $E_{N}$ is then identic with $S_{b}^{\prime}$ as topological vectorspace. Hence $S_{b}^{\prime}$ is nuclear as $E_{N}$ is nuclear ([9] Theorem III.7.4 and corollary). Further $S_{b}^{\prime}$ is Montel as $S_{b}$ is Montel (cf. [2], IV, §3, Prop. 7). 
3. Formal linearlization of the $K D V$-equation. Let $t^{2}$ be the two-dimensional commutative Lie algebra. The $K d V$-equation is defined in the representation

$$
t^{2} \ni(a, b) \stackrel{T}{\mapsto} a T_{0}+b T_{1} \equiv T_{(a, b)} \in F_{s}\left(S_{b}\right),
$$

where

$$
\begin{gathered}
T_{0}=T_{0}^{1}, \quad T_{1}=T_{1}^{1}+T_{1}^{2}, \quad T_{0}^{1}(u)=\partial u, \\
T_{1}^{1}(u)=-\partial^{3} u \text { and } T_{1}^{2}\left(u_{1}, u_{2}\right)=3\left(u_{1} \partial u_{2}+u_{1} \partial u_{1}\right),
\end{gathered}
$$

for $u, u_{1}, u_{2} \in S_{b}$. The representation is formally linearizable [5] on $S_{b}$ into $S_{b}$ if there exists an element $C \in F\left(S_{b}\right)$, with $C^{1}$ continuously invertible and

$$
T_{X} C=C * T_{X}^{1}, \quad \forall X \in t^{2} .
$$

( $C$ takes then the space where the linear equation is defined into the space where the non-linear equation is defined.) The $n$th order of equation (3.2) reads

(3.3) $T_{X}^{1} C^{n}-C^{n} * T_{X}^{1}=-T_{X}^{2}\left(\sum_{1 \leq p \leq n-1} C^{p} \otimes C^{n-p}\right) \sigma_{n}, \forall X \in t^{2}$.

For $X=(1,0)$ we get explicitly

$$
\begin{aligned}
\partial C^{n}\left(\varphi_{2} \otimes \cdots \otimes \varphi_{n}\right) & \\
& =\sum_{1 \leq i \leq n} C^{n}\left(\varphi_{1} \otimes \cdots \otimes \partial \varphi_{i} \otimes \cdots \otimes \varphi_{n}\right), \quad n \geq 1 .
\end{aligned}
$$

LEMMA 3.1. If $C^{n} \in \mathcal{E}_{n}^{s}\left(S_{b}\right)$ and $C^{n}$ satisfies (3.4) then there exists a unique $F^{n} \in S^{\prime}\left(\mathbf{R}^{n}\right)$, symmetric with $\left.\left.\operatorname{supp} F_{n} \subset X^{n}\right]-\infty, k\right]$ for some $k \geq 0$ and

$$
C^{n}\left(\varphi_{1} \otimes \cdots \otimes \varphi_{n}\right)=\left[\check{F}^{n} \circledast\left(\varphi_{1} \otimes \cdots \otimes \varphi_{n}\right)\right] \circ i_{n}
$$

where $i_{n}(x)=(x, \ldots, x)$ (n-times), denotes space inversion and $\circledast$ convolution. Conversely given any $F^{n} \in S^{\prime}\left(\mathbf{R}^{n}\right)$ with $\left.\left.\operatorname{supp} F^{n} \subset X^{n}\right]-\infty, k\right]$, $k \geq 0$, then $C^{n}$ so constructed satisfies equation (3.4).

Proof. If $C^{n} \in \mathcal{L}_{n}^{s}\left(S_{b}\right)$, then

$$
F^{n}\left(\phi_{1} \otimes \cdots \otimes \phi_{n}\right)=\left[C^{n}\left(\phi_{1} \otimes \cdots \otimes \phi_{n}\right)(0)\right.
$$


defines a symmetric element $F_{n} \in S^{\prime}\left(\mathbf{R}^{n}\right)$ and $\left.\left.\operatorname{supp} F^{n} \subset X^{n}\right]-\infty, k\right]$ for some $k \geq 0$ (Proposition 2.2). Let $\tau_{a}$ denote the operator of translation by $a$ on $S_{b}$. Then (3.4) gives

$$
\tau_{a} C^{n}\left(\varphi_{1} \otimes \cdots \otimes \varphi_{n}\right)=F^{n}\left(\tau_{a} \varphi_{1} \otimes \cdots \otimes \tau_{a} \varphi_{n}\right) \quad \forall a \in \mathbf{R}, \varphi_{i} \in S_{b} .
$$

Hence

$$
\begin{aligned}
{\left[C^{n}\left(\varphi_{1} \otimes \cdots \otimes \varphi_{n}\right)\right](x) } & =F^{n}\left(\tau_{x} \varphi_{1} \otimes \cdots \otimes \tau_{x} \varphi_{n}\right) \\
& \equiv\left[\check{F}^{n} \circledast\left(\varphi_{1} \otimes \cdots \varphi_{n}\right)\right] \circ i_{n}(x) .
\end{aligned}
$$

The converse is obvious.

We next prove that a cohomology space $H^{0}\left(t^{2}, \mathcal{L}_{n}^{s}\left(S_{b}\right)\right)=0$.

Proposition 3.2. For $n \geq 2$, the equation

$$
T_{X}^{1} C^{n}-C^{n} * T_{X}^{1}=0, \quad \forall x \in t^{2}
$$

has the unique solution $C^{n}=0$ in $\mathcal{L}_{n}^{s}\left(S_{b}\right)$.

Proof. By Lemma 3.1 each solution $C^{n}$ has the form

$$
C^{n}\left(\varphi_{1} \otimes \cdots \otimes \varphi_{n}\right)=\left[\check{F}^{n} \circledast\left(\varphi_{1} \otimes \cdots \otimes \varphi_{n}\right)\right] \circ i_{n} \quad(F \text { symmetric })
$$

Take $X=(0,1)$. Then, we get

$$
-\partial^{3} C^{n}\left(\varphi_{1} \otimes \cdots \otimes \varphi_{n}\right)+\sum_{1 \leq i \leq n} C^{n}\left(\varphi_{1} \otimes \cdots \otimes \partial^{3} \varphi_{i} \otimes \cdots \otimes \varphi_{n}\right)=0 .
$$

Thus

$$
\left[\left(\partial_{1}+\cdots+\partial_{n}\right)^{3}-\left(\partial_{1}^{3}+\cdots+\partial_{n}^{3}\right)\right] F^{n}=0
$$

After Fourier transformation ${ }^{1}$

$$
P_{n} \tilde{F}^{n}=0, \quad P_{n}\left(k_{1}, \ldots, k_{n}\right)=\left(k_{1}+\cdots+k_{n}\right)^{3}-\left(k_{1}^{3}+\cdots+k_{n}^{3}\right),
$$

where $F^{n}$ is analytic in the domain $\pi_{+}^{n}=\left\{k \mid \operatorname{Re} k_{i}>0,1 \leq i \leq n\right\}$. (Proposition 2.2). But $P_{n} \neq 0$ for $n \geq 2$ and the ring of analytic functions on $\pi_{+}^{n}$ is an integral domain so $F^{n}=0$ is the only solution.

COROLlaRY 3.3. Equation (3.2) has at most one solution for a given $C^{1}$.

\footnotetext{
${ }^{1}$ Fourier transformation in $S\left(\mathbf{R}^{n}\right)$ is defined by $\tilde{f}(k)=(2 \pi)^{-n / 2} \int_{\mathbf{R}^{n}} d x e^{-i k x} f(x)$.
} 
To solve equation (3.3) we introduce the holomorphic functions $\tilde{G}^{n}$ : $\pi_{+}^{n} \rightarrow \mathbf{C}$,

$$
\begin{aligned}
& \quad \tilde{G}^{1}(k)=i k(2 \pi)^{-1 / 2}, \quad \tilde{G}^{2}\left(k_{2}, k_{2}\right)=(2 \pi)^{-1}, \\
& \tilde{G}^{n}\left(k_{1}, \ldots, k_{n}\right) \\
& \quad=\frac{i\left(k_{1}+\cdots+k_{n}\right)}{\left(k_{1}+k_{2}\right) \cdots\left(k_{n-1}+k_{n}\right)}(-i)^{n-1}(2 \pi)^{-n / 2}, \quad n \geq 3 .
\end{aligned}
$$

We introduce also the holomorphic function $\tilde{F}^{n}: \pi_{+}^{n} \rightarrow \mathbf{C}$,

$$
\tilde{F}^{n}\left(k_{1}, \ldots, k_{n}\right)=\frac{1}{n !} \sum_{i \in \mathscr{P}_{n}} \tilde{G}^{n}\left(k_{i_{1}}, \ldots, k_{i_{n}}\right),
$$

where $\mathscr{P}_{n}$ is the group of permutations of $n$ elements. The holomorphic functions $\tilde{G}^{n}$ (resp. $\tilde{F}^{n}$ ) define uniquely (cf. [10]), by Fourier-Laplace transformation, distributions $G^{n}$ (resp. $\left.F^{n}\right) \in S^{\prime}\left(\mathbf{R}^{n}\right)$ with supp $G^{n}$ (resp. $\left.\left.\left.F^{n}\right) \in x^{n}\right]-\infty, k\right]$ for some $k \geq 0$. Lemma 3.1 can now be applied to construct an element $C \in F\left(S_{b}\right)$ (resp. $C_{s} \in F_{s}\left(S_{b}\right)$ ) by the distributions $G^{n}$ (resp. $\left.F^{n}\right)$. The algebraic expressions for $\tilde{G}^{n}$ coincide with them in [8].

Proposition 3.4. $C_{s} \in F_{s}\left(S_{b}\right)$ constructed by (3.5) and (3.6) is the unique translation invariant symmetric formal linearization on $S_{b}$ of the $K d V$-equation, i.e. $T_{X} C_{s}=C_{s} * T_{X}^{1}$ for each $X \in t^{2}$, with $C^{1}=\partial$.

Proof. $C_{s}$ is unique if it exists (Corollary 3.3). By (3.3) and (3.5) the linearization of $T$ by $C_{s}$ is equivalent to

$$
\begin{aligned}
& {\left[\left(\partial_{1}+\cdots+\partial_{n}\right)^{3}-\left(\partial_{1}^{3}+\cdots+\partial_{n}^{3}\right)\right] F^{n}} \\
& \quad=3 \sum_{1 \leq p \leq n-1}\left(\partial_{1}+\cdots+\partial_{n}\right)\left(F^{p} \otimes F^{n-p}\right) \circ \sigma_{n}, \quad n \geq 2 .
\end{aligned}
$$

This is by Fourier-Laplace transformation equivalent to

$$
\begin{aligned}
& {\left[\left(k_{1}+\cdots+k_{n}\right)^{3}-\left(k_{1}^{3}+\cdots+k_{n}^{3}\right)\right] \tilde{F}^{k}\left(k_{1}, \ldots, k_{n}\right)} \\
& \quad=-3\left(k_{1}+\cdots+k_{n}\right) \sum_{1 \leq p \leq n-1} \frac{1}{n !} \sum_{i \in \mathscr{P}_{n}}\left(\tilde{F}^{p} \otimes \tilde{F}^{n-p}\right)\left(k_{i_{1}}, \ldots, k_{i_{n}}\right), \\
& n \geq 2 .
\end{aligned}
$$

$\tilde{F}^{n}$ is a solution of this equation if

$$
\begin{aligned}
& {\left[\left(k_{1}+\cdots+k_{n}\right)^{3}-\left(k_{1}^{3}+\cdots+k_{n}^{3}\right)\right] \tilde{G}^{n}\left(k_{1}, \ldots, k_{n}\right)} \\
& \quad=-3\left(k_{1}+\cdots+k_{n}\right) \sum_{1 \leq p \leq n-1}\left(\tilde{G}^{p} \otimes \tilde{G}^{n-p}\right)\left(k_{1}, \ldots, k_{n}\right), \quad n \geq 2 .
\end{aligned}
$$


Direct substitution as in [8] proves that $\tilde{G}^{n}$ defined by (3.6) satisfies this equation.

The inverse Fourier transformation $G^{n}$ of $\tilde{G}^{n}$, defined by

$$
G^{n}\left(\tilde{\varphi}_{1} \otimes \cdots \otimes \tilde{\varphi}_{n}\right)=\tilde{G}^{n}\left(\varphi_{1} \otimes \cdots \otimes \varphi_{n}\right), \quad \varphi_{1}, \ldots, \varphi_{n} \in S(\mathbf{R}),
$$

is explicitly

$$
\text { (3.7) } \begin{array}{r}
G^{n}\left(u_{1} \otimes \cdots \otimes u_{n}\right)=(-1)^{n} \int_{-\infty}^{0} d y_{1} \\
\cdots \int_{-\infty}^{0} d y_{n-1} u_{1}\left(y_{1}\right) u_{2}\left(y_{1}+y_{2}\right) \\
\cdots u_{n-1}\left(y_{n-2}+y_{n-1}\right) u_{n}\left(y_{n-1}\right) .
\end{array}
$$

Let $U$ be the Frechet space of all functions $f \in C^{\infty}\left(\mathbf{R} \times \mathbf{R}^{-}, \mathbf{R}\right)$ for which the seninorms $M_{n}$ are finite:

$$
\text { (3.8) } M_{n}(f)=\sup _{\substack{0 \leq x+y \leq n \\|\alpha| \leq n}}\left|\partial^{\alpha} f(x, y)\right|+\sup _{\substack{x+y \leq 0 \\|\alpha| \leq n}}\left|(1+|x+y|)^{n} \partial^{\alpha} f(x, y)\right| \text {, }
$$

$n=0,1, \ldots$. Here $\partial^{\alpha}=\partial_{1}^{\alpha_{1}} \partial_{2}^{\alpha_{2}}$ and $|\alpha|=\alpha_{1}=\alpha_{2}$. We introduce for $u \in S_{b}$ the commonly used (see [2], cf. [8], [12]) continuous integral operator $\Omega(u): U \rightarrow U$ :

$$
\begin{aligned}
{[\Omega(u) f](x, y) } & =\int_{-\infty}^{0} u(x+y+t) f(x, t) d t, \\
(x, y) & \in \mathbf{R} \times \mathbf{R}^{-}, \quad f \in U .
\end{aligned}
$$

(3.5), (3.7) and (3.9) give the following explicit expression for $\hat{C}^{n}$ in Proposition 3.4:

$$
\left[\hat{C}^{n}(\varphi)\right](x)=\left[\partial_{1} \hat{B}^{n}(\varphi)\right](x, 0)
$$

where

$$
\begin{gathered}
B^{n}\left(\varphi_{1} \otimes \cdots \otimes \varphi_{n}\right)=(-1)^{n} \Omega\left(\varphi_{1}\right) \cdots \Omega\left(\varphi_{n-1}\right) a\left(\varphi_{n}\right), \\
{\left[a\left(\varphi_{n}\right)\right](x, y)=\varphi_{n}(x+y), \quad \varphi_{1}, \ldots, \varphi_{n} \in S_{b} .}
\end{gathered}
$$

(3.9) and (3.11) give

$$
\hat{B}(\varphi)+\Omega(\varphi) \hat{B}(\varphi)+a(\varphi)=0, \quad B=\sum_{n \geq 1} B^{n} .
$$

The inverse $A=C^{-1}$ is needed for solving the Cauchy problem for the $K d V$ equation. As is seen directly from (3.12), the power series $B$ is easily inverted (on its image):

If $B(\phi)=\psi$, then $\phi=P(\psi)$, where

$$
\hat{P}(\psi)+\mathscr{B}(\psi) \hat{P}(\psi)+\psi(\cdot, 0)=0
$$


and

$$
[\Re(f) u](x)=\int_{-\infty}^{0} f(x, t) u(x+t) d t
$$

defines a continuous integral operator $\Re(f): S_{b} \rightarrow S_{b}$ for each $f \in U$.

It seems difficult to invert $C$ directly. However there is a unique power series $Q_{s} \in F_{s}\left(S_{b}, U\right)$ such that $\hat{Q}_{s} \circ \hat{C}_{s}=\hat{B}_{s}$. To find the inverse of $C$ we first construct a $Q \in F\left(S_{b}, U\right)$. The expressions (3.10) and (3.11) give

$$
\sum_{\substack{1 \leq k \leq N \\ n_{1}+\cdots+n_{k}=N}} Q^{k} \circ\left(\hat{C}^{n_{1}} \otimes \cdots \otimes \hat{C}^{n_{k}}\right)=B^{N}, \quad N=1,2, \ldots
$$

Multiplication with $\Omega(\varphi)$ gives by (3.11)

$$
\begin{aligned}
- & \sum_{\substack{1 \leq k \leq N \\
n_{1}+\cdots+n_{k}=N}} \Omega(\varphi) Q^{k}\left(\hat{C}^{n_{1}}(\varphi) \otimes \cdots \otimes \hat{C}^{n_{k}}(\varphi)\right) \\
= & \sum_{\substack{1 \leq k \leq N+1 \\
n_{1}+\cdots+n_{k}=N+1}} Q^{k}\left(\hat{C}^{n_{1}}(\varphi) \otimes \cdots \otimes \hat{C}^{n_{k}}(\varphi)\right) .
\end{aligned}
$$

Finally by (3.10) and (3.11)

$Q^{N+1}(\partial \varphi \otimes \cdots \otimes \partial \varphi)$

$$
\begin{aligned}
& =\sum_{\substack{1 \leq k \leq N \\
n_{1}+\cdots+n_{k}=N}} \Omega(\varphi) Q^{k}\left(\left[\partial \Omega^{n_{1}-1}(\varphi) a(\varphi)\right](\cdot, 0) \otimes \cdots\right. \\
& \left.\otimes\left[\partial \Omega^{n_{k}-1}(\varphi) a(\varphi)\right](\cdot, 0)\right) \\
& -\sum_{\substack{1 \leq k \leq N, m_{l} \geq 1 \\
m_{1}+\cdots+m_{k}=N+1}} Q^{k}\left(\left[\partial \Omega^{m_{1}-1}(\varphi) a(\varphi)\right](\cdot, 0) \otimes \cdots\right. \\
& \left.\otimes\left[\partial \Omega^{m_{k}-1}(\varphi) a(\varphi)\right](\cdot, 0)\right) .
\end{aligned}
$$

A lengthy but straight forward calculation gives that

$$
\hat{Q}^{N}(\partial \varphi)=\mathbb{Q}^{N-1}(\partial \varphi) a(\varphi), \quad N=1,2, \ldots,
$$

where

$$
[\mathbb{Q}(u) f](x, y)=\int_{y}^{0} d z \int_{-\infty}^{x+y-z} d t u(t) f(t, z)
$$

defines for each $u \in S_{b}$ a continuous operator $U \rightarrow U$. (The operator $\mathbb{Q}$ is well-known, cf. [4].) It follows then that

$$
\hat{Q}^{N}(\varphi)=\mathscr{Q}(\varphi)^{N-1} a\left(\partial^{-1} \varphi\right), \quad N=1,2, \ldots,
$$


and that $\hat{Q}(\phi)$ satisfies the integral equation

$$
\hat{Q}(\varphi)-\mathbb{Q}(\varphi) \hat{Q}(\varphi)=a\left(\partial^{-1} \varphi\right) \text {. }
$$

4. Convergence properties of the formal power series. We study in this paragraph the existence and the properties of the functions defined by the formal power series $Q, P, B$ and by them $A$ and $A^{-1} \cdot \hat{Q}, \hat{P}, \hat{B}$ are uniquely defined by the integral equations (3.17), (3.13) resp. (3.12):

$$
\hat{Q}(\varphi)-\mathscr{Q}(\varphi) \hat{Q}(\varphi)=a\left(\partial^{-1} \varphi\right)
$$$$
Q \in F\left(S_{b}, U\right), \varphi \in S_{b},
$$

(4.2) $\hat{P}(f)+\mathscr{B}(f) \hat{P}(f)=-f(\cdot, 0)$, $P \in F\left(U, S_{b}\right), f \in U$,

$$
\hat{B}(\varphi)+\Omega(\varphi) \hat{B}(\varphi)=-a(\varphi)
$$$$
B \in F\left(S_{b}, U\right), \varphi \in S_{b} \text {. }
$$

Proposition 4.1. The formal power series $Q$ (resp. $P$ ) converges to an entire function $\hat{Q}: S_{b} \rightarrow U$ (resp. $\hat{P}: U \rightarrow S_{b}$ ). $\hat{Q}($ resp. $\hat{P}$ ) is the unique solution of (4.1) (resp. (4.2)).

Proof. Using that

$$
\left[\partial_{1} \mathscr{Q}(\varphi) f\right](x, y)=\int_{y}^{0} d y_{1} \varphi\left(x+y-y_{1}\right) f\left(x+y-y_{1}, y_{1}\right)
$$

and that

$$
\left[\left(\partial_{1}-\partial_{2}\right) \mathcal{Q}(\varphi) f\right](x, y)=\int_{-\infty}^{x} d x_{1} \varphi\left(x_{1}\right) f\left(x_{1}, y\right)
$$

one deduces the existence of seminorms $p_{0} \leq p_{1} \leq \cdots$ for each given $K \geq 0$ such for $\alpha_{1} \geq 1$ :

$$
\begin{aligned}
\sup _{|\alpha| \leq N}\left|\partial^{\alpha} \mathscr{Q}(\varphi) f\right|(x, y) \leq p_{N}(\varphi) & \sup _{\substack{|\alpha| \leq N \\
\alpha_{1} \neq N \\
\left(x_{1}, y_{1}\right) \in E(x, y)}}\left|\partial^{\alpha} f\left(x_{1}, y_{1}\right)\right|, \\
& \forall(x, y) \in]-\infty, K] \times \mathbf{R}^{-},
\end{aligned}
$$

and for $\alpha_{1}=0$ :

$$
\begin{array}{r}
\sup _{0 \leq \alpha_{2} \leq N}\left|\partial_{2}^{\alpha_{2}} \mathscr{Q}(\varphi) f\right|(x, y) \leq p_{N}(\varphi) \sup _{\substack{|\alpha| \leq N-1 \\
\left(x_{1}, y_{1}\right) \in E(x, y)}}\left|\partial^{\alpha} f\left(x_{1}, y_{1}\right)\right| \\
\forall(x, y) \in]-\infty, K] \times \mathbf{R}^{-}
\end{array}
$$


where $E(x, y)=\left\{\left(x_{1}, y_{1}\right) \in \mathbf{R} \times \mathbf{R}^{-} \mid x_{1}+y_{1} \leq x+y\right\}$. We have here used the inequality (cf. [12])

(4.6) $|\mathcal{Q}(\varphi) f|(x, y) \leq \pi \int_{-\infty}^{x} d t\left(1+t^{2}\right)|\varphi(t)| \sup _{\left(x_{1}, y_{1}\right) \in E(x, y)}\left|f\left(x_{1}, y_{1}\right)\right|$.

Denote

$$
\eta_{x}(\varphi)=\pi \int_{-\infty}^{x} d t\left(1+t^{2}\right)|\varphi(t)|
$$

The explicit form of $\mathbb{Q}(\phi)$ and (4.4), (4.5) (4.6) give for $M \geq N+1$ and $(x, y) \in]-\infty, K] \times \mathbf{R}^{-}$:

$$
\begin{aligned}
& \sup _{|\alpha| \leq N}\left|\partial^{\alpha} \mathbb{Q}^{M-1}(\varphi) a\left(\partial^{-1} \varphi\right)\right|(x, y) \\
& \quad \leq[(M-1-N) !]^{-1} p_{N}(\varphi) \eta_{x}(\varphi)^{M-1-N} \sup _{t \leq x+y}\left|\partial^{-1} \varphi(t)\right| .
\end{aligned}
$$

Finally we find that (see (3.8), (3.16))

$$
M_{N}\left(\hat{Q}^{n}(\varphi)\right) \leq p_{N}(\varphi)^{N+1} \eta_{k}(\varphi)^{n-1-N}[(n-1-N) !]^{-1},
$$

where $n \geq N+1$. $p_{N}$ has (for convenience) been chosen sufficiently large and $K \geq N$. (4.8) proves that the series $\hat{Q}(\phi)$ converges for each $\phi \in S_{b}$. Hence $\hat{Q}: S_{b} \rightarrow U$ is entire analytic.

To prove the second statement, we first remark that

$$
[\Re(\psi) \varphi](x)=\int_{-\infty}^{x} d t \psi(x, t-x) \varphi(t)
$$

gives

$$
[\partial \mathscr{B}(\psi) \varphi](x)=\psi(x, 0) \varphi(x)+\Re\left(\left(\partial_{1}-\partial_{2}\right) \psi\right) \varphi .
$$

Formula (4.9) and (4.10) give the estimate, for $n \geq N+1$,

(4.11) $\left|\partial^{N} \mathfrak{B}^{n-1}(\psi) \varphi\right|(x)$

$$
\leq q_{N}(\psi)^{N}[(n-1-N) !]^{-1}\left(\int_{-\infty}^{x} d t|\psi(x, t-x)|\right)^{n-1-N} \sup _{t \leq x}|\varphi(t)|,
$$

where $q_{N}$ is a sufficiently large seminorm on $U$. Then for $p_{N}$ sufficiently large

$$
\left\|\mathscr{B}^{n-1}(\psi) \varphi\right\|_{N} \leq p_{N}(\psi)^{n-1}\|\varphi\|_{N}[(n-1-N) !]^{-1}
$$


Thus, once more if $p_{N}$ is sufficiently large

$$
\left\|\hat{P}^{n}(\psi)\right\|_{N} \leq p_{N}(\psi)^{n}[(n-1-N) !]^{-1},
$$

which proves that $\hat{P}$ is entire analytic.

Corollary 4.2. Denote $A=C^{-1} \in F\left(S_{b}\right)$. Then $\hat{A}$ is an entire analytic function.

Proof. $\hat{A}=\hat{P} \circ \hat{Q}$ is entire analytic by Proposition 4.1 .

We now turn to the question of the existence of $\hat{A}^{-1}$. We prove the convergence of $C=A^{-1}$ and find the maximal connected domain of analyticity (containing $u=0$ ) for $\hat{C}$.

Introduce the operator (see [4]) $\left.\left.\left.\left.\Omega_{x}(u): L^{2}(]-\infty, 0\right]\right) \rightarrow L^{2}(]-\infty, 0\right]\right)$ for $x \in \mathbf{R}, u \in S_{b}$ :

$$
\left[\Omega_{x}(u) h\right](y)=\int_{-\infty}^{0} u(x+y+t) h(t) d t .
$$

$\Omega_{x}(u)$ is a self-adjoint Hilbert-Schmidt operator with

$$
\left\|\Omega_{x}(u)\right\|_{\text {H.S. }} \leq\left(\frac{\pi}{2}\right)^{1 / 2} \sup _{t \leq 0}\left|\left(1+t^{2}\right) u(x+t)\right| .
$$

Let $\sigma\left(\Omega_{x}(u)\right)$ be the spectrum of $\Omega_{x}(u)$, let $U(n), n=0,1, \ldots$, be the factorspace of $U$ and the closed subspace of functions $f$ with supp $f \subset$ $\left[n, \infty\left[\times \mathbf{R}^{-} . B \in F\left(S_{b}, U\right)\right.\right.$ defines an element in $F(S(n), U(n))$ which also will be denoted $B$. The seminorms on $U(n)$ induced by the complete set of seminorms (3.8) on $U$ will be denoted $M_{N}^{n}, N=0,1, \ldots$

Proposition 4.3. For each $n \in \mathbf{N}, B$ defines an analytic function $\hat{B}$ : $O_{n} \supset S(n) \rightarrow U(n)$, where $O_{n}$ is the set of all $u \in S(n)$ such that $\Omega_{x}(u)>-I$, $\forall x \in]-\infty, n] . O_{n}$ is open in $S(n)$ and

$$
[\hat{B}(u)](x, y)=-\left[\left(I+\Omega_{x}(u)\right)^{-1}[a(u)](x, \cdot)\right](y) .
$$

(Analytic means here convergence on a neighbourhood of each point in $O_{n}$ ).

Proof. Let $u \in O_{n}$. Then by estimate (4.14) there exists an neighbourhood $V$ of $u$ such that, for each $v \in V$ and $x \in]-\infty, n], \sigma\left(\Omega_{x}(v)\right) \subset$ $\left[-1+\varepsilon, \infty\left[\right.\right.$ for some $0<\varepsilon$. Hence $O_{n}$ is open in $S(n)$. 
It follows from Lemma III.9 in [12], (where it is only used that $\sigma\left(\Omega_{x}(u)\right) \subset[-1+\varepsilon, \infty[$ for each $\left.x \in]-\infty, n]\right)$, that $\hat{B}(u) \in U(n)$ and that $\left[I+\Omega_{x}(u)\right]^{-1} f(x, \cdot)$ defines an element in $U(n)$ for each $f \in U(n)$. Let $u, u+\phi \in O_{n}$. Then at the point $u+\phi$, the r.h.s. of (4.15) can be written

$$
\begin{aligned}
-(I+\Omega(u+\varphi))^{-1} a(u+\varphi) & \\
& =-\left[I+\Omega(\varphi)(I+\Omega(u))^{-1}\right]^{-1}(I+\Omega(u))^{-1} a(u+\varphi) .
\end{aligned}
$$

We prove the convergence of the series

$$
\sum_{0 \leq k}(-1)^{k}\left[\Omega(\varphi)(I+\Omega(u))^{-1}\right]^{k}(I+\Omega(u))^{-1} a(u+\varphi)
$$

in $U(n)$ for all $\phi$ in some $S(n)$ neighbourhood of $u$. Let $\phi_{1}, \ldots, \phi_{k-1} \in$ $S(n), u_{1} \ldots, u_{k} \in O_{n}, k \geq 2, f \in U(n)$ and define the functions $F^{k}$ : $S(n)^{k-1} \times O_{n}^{k} \times U(n) \rightarrow U(n):$

(4.17) $F^{k}\left(\varphi_{1}, \ldots, \varphi_{k-1} ; u_{1}, \ldots, u_{k-1} ; f\right)$

$$
\begin{aligned}
= & (-1)^{k} \Omega\left(\varphi_{1}\right)\left(I+\Omega\left(u_{1}\right)\right)^{-1} \Omega\left(\varphi_{2}\right)\left(I+\Omega\left(u_{2}\right)\right)^{-1} \\
& \cdots \Omega\left(\varphi_{k-1}\right)\left(I+\Omega\left(u_{k-1}\right)\right)^{-1} f .
\end{aligned}
$$

The following estimates are obtained directly from formula (3.9) and the identity $-(I+\Omega(u))^{-1} f-\Omega(u)(I+\Omega(u))^{-1} f+f=0, u \in O_{n}, f \in U(n)$ :

$$
\begin{aligned}
& \sup _{\substack{x \leq n \\
y \leq 0}}\left|(1+|x|+|y|)^{N}[\Omega(\varphi) f](x, y)\right| \\
& \quad \leq p_{N}(\varphi) \sup _{\substack{x \leq n \\
y \leq 0}}|f(x, y)|, \quad \forall \varphi \in S(n), f \in U(n), N \in \mathbf{N},
\end{aligned}
$$

where $p_{0} \leq \cdots \leq p_{N} \leq \cdots$ are sufficiently large seminorms on $S(n)$ and

$$
\begin{aligned}
\mid f(x, y)- & {\left[(I+\Omega(u))^{-1} f\right](x, y) \mid } \\
\leq & \left\|\left(I+\Omega_{x}(u)\right)^{-1}\right\|_{L^{2}} \cdot \sup _{t \leq 0}\left|(1-t)^{2} u(x+y+t)\right| \\
& \cdot \sup _{t \leq 0}\left|(1-t)^{2} f(x, t)\right| .
\end{aligned}
$$

(4.19) gives:

(4.20) $\sup _{x \leq n}\left|\left[(I+\Omega(u))^{-1} f\right](x, y)\right| \leq C_{u} \sup _{t \leq 0}\left|(1-t)^{2} f(x, t)\right|$, $x \leq n$
$y \leq 0$ 
where (for given $n$ ) $C_{u}$ is a positive constant depending on $u \in O_{n}$. (4.18) and (4.20) give for some sufficiently large seminorm $q$ on $S(n)$ :

$$
\text { (4.21) } \begin{aligned}
\sup _{\substack{x \leq n \\
y \leq 0}}\left|\left[(I+\Omega(u))^{-1} \Omega(\varphi) f\right](x, y)\right| \\
\leq C_{u} q(\varphi) \sup _{\substack{x \leq n \\
y \leq 0}}|f(x, y)|, \quad \forall u \in O_{n}, \varphi \in S(n), f \in U(n) .
\end{aligned}
$$

By (4.18) (4.21) and then (4.20) an estimation for $F^{k}$ is obtained:

$$
\text { (4.22) } \begin{aligned}
\sup _{\substack{x \leq n \\
y \leq 0}}\left|(1+|x|+|y|)^{N}\left[F^{k}\left(\varphi_{1}, \ldots, \varphi_{k-1} ; u_{1}, \ldots, u_{k-1} ; f\right)\right](x, y)\right| \\
\quad \leq p_{N}\left(\varphi_{1}\right) C_{u_{1}} \cdots C_{u_{k-1}} q\left(\varphi_{2}\right) \cdots q\left(\varphi_{k-1}\right) \sup _{\substack{t \leq 0 \\
x \leq n}}\left|(1-t)^{2} f(x, t)\right| .
\end{aligned}
$$

The identity (for a given $x \in]-\infty, n]$ ) for $\varepsilon \leq n-x$

$$
-\left(I+\Omega_{x+\varepsilon}(u)\right)^{-1}-\Omega_{x+\varepsilon}(u)\left(I+\Omega_{x+\varepsilon}(u)\right)^{-1}+I=0
$$

gives

(4.23) $\left.\frac{d}{d \varepsilon}\left(I+\Omega_{x+\varepsilon}(u)\right)^{-1}\right|_{\varepsilon=0}=\left(I+\Omega_{x}(u)\right)^{-1} \Omega_{x}(\partial u)\left(I+\Omega_{x}(u)\right)^{-1}$

(This is well-defined in $U(n)$, cf. [9].)

Explicit expressions for the derivatives of $F^{k}(\varphi ; u ; f)$ are obtained from (4.17), (3.9) and (4.23):

$$
\begin{aligned}
& \partial_{1} F^{k}\left(\varphi_{1}, \ldots, \varphi_{k-1} ; u_{1}, \ldots, u_{k-1} ; f\right) \\
& =\sum_{1 \leq i \leq k-1}\left\{F^{k}\left(\varphi_{1}, \ldots, \partial \varphi_{i}, \ldots, \varphi_{k-1} ; u_{1}, \ldots, u_{k-1} ; f\right)\right. \\
& \quad+F^{k+1}\left(\varphi_{1}, \ldots, \varphi_{i}, \partial u_{i}, \varphi_{i+1}, \ldots, \varphi_{k-1} ;\right. \\
& \left.\left.\quad u_{1}, \ldots, u_{i}, u_{i}, u_{i+1}, \ldots, u_{k-1} ; f\right)\right\} \\
& \quad+F^{k}\left(\varphi_{1}, \ldots, \varphi_{k-1} ; u_{1}, \ldots, u_{k-1} ; \partial_{1} f\right)
\end{aligned}
$$

and

$$
\begin{aligned}
\partial_{2} F^{k}\left(\varphi_{1}, \ldots, \varphi_{k-1} ; u_{1}, \ldots, u_{k-1} ; f\right) \\
\quad=F^{k}\left(\partial \varphi_{1}, \varphi_{2}, \ldots, \varphi_{k-1} ; u_{1}, \ldots, u_{k-1} ; f\right)
\end{aligned}
$$


(4.22), (4.24) and (4.25) give the estimate for $k \geq N+1\left(q_{N}\right.$ is a sufficiently large seminorm):

$$
\begin{aligned}
M_{N}\left(F^{k}(\varphi, \ldots, \varphi ; u, \ldots, u ; f)\right. \\
\leq(2(k+N)-1)^{N} q_{N}(\varphi)^{N}\left(C_{u}\right)^{k-N-1} \\
\quad \times \sup _{\substack{x \leq n \\
t \leq 0 \\
0 \leq \alpha \leq N}}\left|(1-t)^{2} \partial_{1}^{\alpha} f(x, t)\right| q(\varphi)^{k-N-1} .
\end{aligned}
$$

Thus, for a given $u \in O_{n}$ the series

$$
\sum_{k \geq 2} F^{k}(\varphi, \ldots, \varphi ; u, \ldots, u ; f)
$$

converges for all $f \in U(n)$ and all $\phi \in S(n)$ with $q(\phi) C_{u}<1 . f=$ $(I+\Omega(u))^{-1} a(u+\phi)$ is linear (and continuous) in $\phi$, which proves that the series (4.16) converges on a neighbourhood of $u$ in $S(n)$.

Corollary 4.4. The mapping $O_{n} \ni u \mapsto\left[\partial_{t} \hat{B}(u)\right](\cdot, \circ) \equiv \hat{C}(u) \in$ $S(n)$ is analytic and this mapping composed with $\hat{A}$ is $\mathrm{id}_{O_{n}}$.

Proof. The first statement is a trivial consequence of Proposition 4.3. Secondly denote $\hat{F}: O_{n} \rightarrow S(n)$ the composite map $\hat{A} \circ \hat{C} . \hat{F}$ is analytic (Corollary 4.2 and Proposition 4.3) and the formal power series $F$ on $S(n)$ is the identity $(\S 3)$.

REMARK 4.5. Let $\left\{u_{l}\right\}_{l=1}^{\infty} \subset O_{n}$ be a convergent sequence in $S(n)$ with limit $v$, such that $-1 \in \sigma\left(\Omega_{x}(v)\right)$ for some $\left.\left.x \in\right]-\infty, n\right]$. Then by (4.15) $\hat{B}\left(u_{l}\right)$ will likely (if there is no cancellation) develop singularities as $l \rightarrow \infty$, and $v$ would not be in $\hat{A}[S(n)]$. Further if for $u \in S(n),-1 \notin$ $\sigma\left(\Omega_{x}(u)\right)$ for $\left.\left.x \in\right]-\infty, n\right]$, then by the continuity of $x \rightarrow \Omega_{x}(u), \sigma\left(\Omega_{x}(u)\right)$ $\subset[-1+\varepsilon, \infty[$ for each $x \in]-\infty, n]$ for some $\varepsilon>0$. Thus by Corollary 4.4. it seems reasonable to try to prove that $\Omega_{x}(u)>-I$ for each $u \in$ $\hat{A}[S(n)]$ and $x \in]-\infty, n]$. The result is well-known, (see [4]).

Proposition 4.6. The image $\hat{A}\left[S_{b}\right]$ is exactly the subset $O \subset S_{b}$, where $O$ is the projective limit of $O_{n}, n \in \mathbf{N}$, i.e. $u \in O$ iff

$$
\Omega_{x}(u)>I \quad \forall x \in \mathbf{R} .
$$

Proof. For given $x \in \mathbf{R}$ choose $n>x$. Then as $\hat{A}: S_{b} \rightarrow S_{b}$ defines a mapping $\hat{A}: S(n) \rightarrow S(n)$

$$
[\hat{A}(u)](x)=\left[\hat{A}\left(\theta_{n+1} u\right)\right](x), \quad \forall u \in S_{b},
$$


where $\theta_{n+1} \in C^{\infty}(\mathbf{R}) . \theta_{n+1}(t)=1$ for $\left.\left.t \in\right]-\infty, n\right]$ and $\theta_{n+1}(t)=0$ for

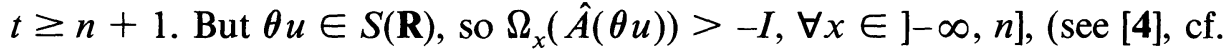
[12]).

REMARK 4.7. The condition (4.27) resembles to that obtained in linearizing Burger's equation in [13].

5. Solution of the non-linear initial value problem and superposition of solutions. Corollaries 4.2 and 4.4 reduce the solution of the $K d V$ equation in $S_{b}$ :

$$
\frac{d}{d t} u(t)=-\partial^{3} u(t)+6 u(t) \partial u(t), \quad u(0)=u_{0} \in S_{b}
$$

to the linear equation

$$
\frac{d}{d t} v(t)=-\partial^{3} v(t), \quad v(0)=v_{0}=\hat{A}\left(u_{0}\right) \in 0
$$

together with the condition in Proposition 4.6. But before stating formally the result we prove the following lemma:

LEMMA 5.1. Let $V$ be a $n$-dimensional $C^{\infty}$ differentiable manifold and $F$ : $V \rightarrow O$ a $C^{\infty}$ mapping then

$$
f=\hat{A}^{-1} \circ F: V \rightarrow S_{b} \text { is } C^{\infty} .
$$

Proof. The mapping $\left.V \ni \xi \mapsto[F(\xi)]\right|_{-\infty, n]} \in O_{n}$ is $C^{\infty}$ and $\hat{A}^{-1}: O_{n} \rightarrow$ $S(n)$ is analytic (Corollary 4.4). Then the mapping $V \ni \xi \mapsto$ $\left[p_{n} \circ \hat{A}^{-1} \circ F\right](\xi) \in S(n)$ is $C^{\infty}$, where $p_{n}: S_{b} \rightarrow S(n)$ is the canonical projection. Thus $V \ni \xi \mapsto\left[\hat{A}^{-1} \circ F\right](\xi) \in S_{b}$ is $C^{\infty}$ as $S_{b}=\lim _{\leftarrow} S(n)$.

Proposition 5.2. Let $u_{0} \in S_{b}$. Then equation (5.1) has a $C^{1}$ solution for $t \in] a_{1}, a_{2}\left[,-\infty \leq a_{1} \leq 0 \leq a_{2} \leq \infty\right.$, iff the equation (5.2) with $v_{0}=$ $\hat{A}\left(u_{0}\right)$ has a $C^{1}$ solution $v(t) \in O$ for $\left.t \in\right] a_{1}, a_{2}[$. On the interval of existence $u(t)=\hat{A}^{-1}(v(t))$ and $t \mapsto u(t)$ is $C^{\infty}$.

REMARK. Instead of $C^{1}$ solutions one can write $C^{0}$ solution of the integrated equations.

Proof. $v_{0} \in O$ (Proposition 4.6). There exists a maximal interval ]$b_{1}, b_{2}\left[,-\infty \leq b_{1} \leq 0 \leq b_{2} \leq \infty\right.$, where equation (5.2) has a $C^{1}$ solution in $S_{b}$. Hence the solution $u(t)=\hat{A}^{-1}(v(t))$ of equation (5.1) exists on a maximal interval $t \in] a_{1}, a_{2}\left[, b_{1} \leq a_{1} \leq 0 \leq a_{2} \leq b_{2}\right.$, on which $v(t) \in O$. 
$t \mapsto v(t)$ is $C^{\infty}$ if it is $C^{1}$. Lemma 5.1 gives now the "if part" of the proposition. Conversely if $u(t)$ is a $C^{1}$-solution on a maximal interval of existence ] $a_{1}, a_{2}$ [ then by Corollary (4.2) and Proposition $4.6 \hat{A}(u(t)) \in O$ is a $C^{1}$ solution of equation 5.2 on ] $a_{1}, a_{2}$ [. Further $t \mapsto \hat{A}(u(t))$ is then $C^{\infty}$, so by Lemma $5.1 u(t)$ is $C^{\infty}$.

REMARK 5.3.

(a) $u(t)$ can blow up in finite time ( $t=a$ ) for two reasons:

(i) $v(t) \in$ does not converge in $S_{b}$ as $t \rightarrow a$;

(ii) $v(a) \exists$ in $S_{b}$ but $v(a) \notin O$.

(b) If $u_{0} \in S(\mathbf{R})$ then $u(t) \in S(\mathbf{R}) \forall t \in \mathbf{R}$ as is seen from space-time inversion of equation 5.1 on $S(\mathbf{R})$ and linearization by $A . \hat{A}(S(\mathbf{R}))$ is invariant under the linear evolution, [12].

The non-linear superposition principle is a trivial consequence of the following Corollary:

Corollary 5.4. O is a convex set.

Proof. See Proposition 4.6.

Acknowledgement. I want to thank Professor M. Flato for helpful suggestions and constructive criticism.

\section{REFERENCES}

[1] J. L. Bona and R. Smith, The initial value problem for the Korteweg-de Vries equation, Phil. Trans. Royal Soc. London, (A) 278 (1975), 555-604.

[2] N. Bourbaki, Espaces vectoriels topologiques, Eléments de mathématique, Fascicule XVIII, Livre V, Hermann Paris 1973.

[3] A. Cohen, Existence and regularity for solutions of the Korteweg-de Vries equation, Arch. Rat. Mech. Anal., 71 (1979), 143-175.

[4] L. D. Faddeev, Properties of the S-matrix of the one-dimensional Schrödinger equation, Trudy. Matem. in-ta im. V.A. Steklova, 73 (1964), 314-336.

[5] M. Flato, G. Pinczon and J. Simon, Non-linear representations of Lie groups, Ann. Scient. Ecole Norm. Sup. Paris, 10 (1977), 405-418.

[6] M. Flato and J. Simon, On a linearization program of non-linear field equations, Phys. Lett., 94B (1980), 518-522.

[7] C. S. Gardner, J. M. Green, M. D. Kurskal and R. M. Miura, Method for solving the Koretweg-de Vries equation, Phys. Rev. Lett., 19 (1967), 1095-1097.

[8] R. R. Rosales, Exact solutions of some non-linear evolution equations, Stud. Appl. Math., 59 (1978), 117-151.

[9] H. H. Schaefer, Topological Vector Spaces, Springer-Verlag, New-York, Heidelberg, Berlin 1980. 
[10] L. Schwartz, Théorie des Dsitributions, Hermann 1973,

[11] A. Sjöberg, On the Korteweg-de Vries equation, J. Math. Anal. Appl., 29 (1970), 569-579.

[12] E. Taflin, Dynamical symmetries and conservation laws for the Kortweg-de Vries equation, to appear in Rep. Math. Phys.

[13] Analytic linearization, Hamiltonian formalism and infinite sequences of constants of motion for Burger's equation, Phys. Rev. Lett., 47 (1981), 1425.

Received January 15, 1982. Supported by the Swiss National Science Foundation.

UNIVERSITE DE GENEVE

1211 GENEve 4, SwitZERLAND 


\section{PACIFIC JOURNAL OF MATHEMATICS EDITORS}

Donald BABBITT (Managing Editor)

University of California

Los Angeles, CA 90024

Hugo Rossi

University of Utah

Salt Lake City, UT 84112

C. C. Moore and Arthur Ogus

University of California

Berkeley, CA 94720
J. DugundiI

Department of Mathematics

University of Southern California

Los Angeles, CA 90089-1113

R. Finn and H. SAMELSON

Stanford University

Stanford, CA 94305

ASSOCIATE EDITORS
R. ARENS
E. F. BECKENBACH
B. H. NeUmanN
F. WOLF
K. YosHIDA (1906-1982)

\section{SUPPORTING INSTITUTIONS}

UNIVERSITY OF ARIZONA

UNIVERSITY OF BRITISH COLUMBIA

CALIFORNIA INSTITUTE OF TECHNOLOGY

UNIVERSITY OF CALIFORNIA

MONTANA STATE UNIVERSITY

UNIVERSITY OF NEVADA, RENO

NEW MEXICO STATE UNIVERSITY

OREGON STATE UNIVERSITY
UNIVERSITY OF OREGON

UNIVERSITY OF SOUTHERN CALIFORNIA

STANFORD UNIVERSITY

UNIVERSITY OF HAWAII

UNIVERSITY OF TOKYO

UNIVERSITY OF UTAH

WASHINGTON STATE UNIVERSITY

UNIVERSITY OF WASHINGTON 


\section{Pacific Journal of Mathematics}

\section{Vol. 108, No. $1 \quad$ March, 1983}

Waleed A. Al-Salam and A. Verma, $q$-Konhauser polynomials $\ldots \ldots \ldots \ldots 1$

Alfred David Andrew, The Banach space JT is primary $\ldots \ldots \ldots \ldots \ldots . . .6$

Thomas E. Bengtson, Bessel functions on $P_{n} \ldots \ldots \ldots \ldots \ldots \ldots$

Joaquim Bruna Floris and Francesc Tugores, Free interpolation for

holomorphic functions regular to the boundary $\ldots \ldots \ldots \ldots \ldots \ldots \ldots \ldots$

Peter Dierolf and Susanne Dierolf, Topological properties of the dual pair

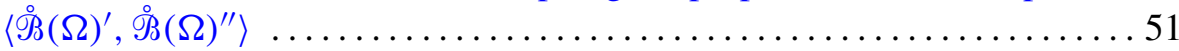

Gerald Arthur Edgar, An ordering for the Banach spaces $\ldots \ldots \ldots \ldots \ldots 83$

Basil Gordon, A proof of the Bender-Knuth conjecture . . . . . . . . . . . . . 99

Harold T. Hodes, A minimal upper bound on a sequence of Turing degrees

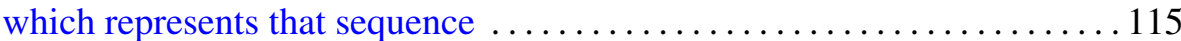

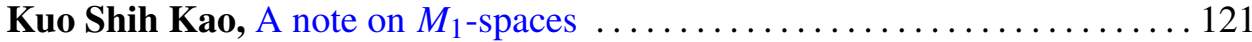

Frank Kost, Topological extensions of product spaces ................ 129

Eva Lowen-Colebunders, On the convergence of closed and compact

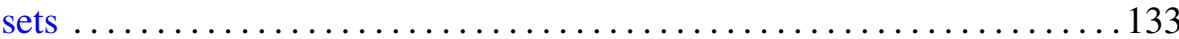

Doron Lubinsky, Divergence of complex rational approximations . . . . . . 141

Warren May and Elias Hanna Toubassi, Endomorphisms of rank one

mixed modules over discrete valuation rings $\ldots \ldots \ldots \ldots \ldots \ldots \ldots \ldots \ldots$

Richard Patrick Morton, The quadratic number fields with cyclic

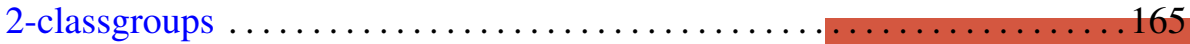

Roderic Murufas, Rank of positive matrix measures . . . . . . . . . . . . 177

Helga Schirmer, Fixed point sets of homotopies . . . . . . . . . . . . . 191

E. Taflin, Analytic linearization of the Korteweg-de Vries equation ........ 203

James Thomas Vance, Jr., $L^{p}$-boundedness of the multiple Hilbert

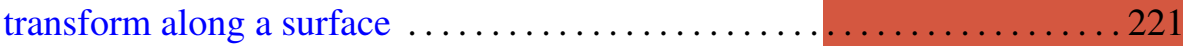

Hiroshi Yamaguchi, A property of some Fourier-Stieltjes transforms . . . . . 243 